\title{
The direct relation between safe hemorrhoidectomy and anal canal anatomy
}

\author{
Theodoros Mariolis-Sapsakos ${ }^{1,2}$, George Skarpas ${ }^{1,2 *}$, Arhontoula Xifara ${ }^{1}$, Maria Zarokosta ${ }^{1,2}$, Theodoros Piperos ${ }^{1,2}$, Menelaos Zoulamoglou ${ }^{1}$ \\ and Ioannis Papapanagiotou ${ }^{1,2}$ \\ ${ }^{1}$ Department of Surgery, General and Oncologic Hospital of Kifissia "Agii Anargiri”, University of Athens, Greece \\ ${ }^{2}$ Anatomy, Histology \& Embryology Laboratory, Nursing School, University of Athens, Greece
}

\begin{abstract}
Hemorrhoids are a common anal disorder that affects both men and women of all ages. One out of ten patients with hemorrhoidal disease, requires surgical treatment. However, hemorrhoidectomy is related to complications that can be present early or late postoperatively. In the present manuscript, the safe surgical technique which emphasizes to the identification of the ligament of Parks (Trietz's muscle) is described. A total of 145 patients with grades III and IV hemorrhoids, underwent Milligan-Morgan or Ferguson's hemorrhoidectomy. The mucosal ligament of Parks was located to all patients and was used as a key anatomical structure through the excision of the hemorrhoids. Its detection guides surgeons during hemorrhoidectomy. Finally, since the ligament of Parks represents a constantly identifiable landmark, it leads to simple and reliable identification of the internal sphincter and minimizes the potentiality of postoperative complications. Hereby, surgeons' thorough knowledge of the anatomy of the anal canal is a cornerstone of safe hemorrhoidectomy.
\end{abstract}

\section{Introduction}

Hemorrhoidal disease is a common anal disorder that affects both male and female patients of all ages [1-3]. Hemorrhoids are defined as the enlargement and displacement of the anal cushions [4] that lead to a variety of symptoms that subsequently determine the appropriate therapeutical approach. However, one out of ten patients requires surgical treatment [5]. Unfortunately, conventional hemorrhoidectomy is closely related to complications that can be present early or late postoperatively [1]. In the present manuscript, after the successful performance of hemorrhoidectomy on 145 patients with grades III and IV hemorrhoids, the safe surgical technique which emphasizes to the identification of the key anatomical structure of the ligament of Parks (Trietz's muscle) is described.

\section{Methods}

During the period 2000-2013, 76 men and 69 women with ages from 27 to 85 proceeded to our institution.

From this total of 145 patients, 73 patients (50.3\%) had grade III and 72 (49.7\%) had grade IV hemorrhoids. None of the patients had severe comorbidities that could exclude the performance of conventional hemorrhoidectomy under general anesthesia.

Before the operation, a phosphate enema is administrated to the patient for clearance and better approach to the operative field. Additionally, one dose of metronidazole is administrated as antibiotic prophylaxis, two hours before the onset of the surgery. One more dose of the antibiotic is administrated to the patient postoperatively as well. Patients underwent Milligan-Morgan or Ferguson's hemorrhoidectomy, placed in lithotomy position, knees flexed (Figure 1).

\section{Technique presentation}

Milligan-Morgan procedure: Based on Milligan- Morgan technique, at first, three Foerster clamps were placed on the peri-anal skin, along the axis of the hemorrhoidal pedicles (3, 7, 11 o'clock). Then, three other Foerster clamps were placed at the same points, at the dentate line of the anal canal. A solution of xylocaine with $1 \%$ epinephrine was administrated subcutaneously, at the level of each hemorrhoidal pedicle, below the dentate line in order to minimize bleeding during the dissection. Then, three Kelly clamps were placed on the rectal mucosa at the same points $(3,7,11$ o'clock).

All pedicles were meticulously dissected and only a mucocutaneous bridge was maintained between each pedicle. Hence, a cutaneous triangle of exposure was created (Figure 2). The inside summit of this triangle is defined by the second Foerster clamp. The dissection continued progressively and in the submucosal level, below the level of the dentate line, the mucosal ligament of Parks was identified (Figure 3). The Parks' ligament (Trietz's muscle) passes through the internal sphincter muscle and fixes the submucosal tissues to the conjoined longitudinal muscle. This anatomic landmark that is consisted of mucosal suspensory ligament and anal submucosal muscle was promptly divided and below it, the muscular fibers of the internal sphincter were revealed. In fact, this ligament was used as the lateral border of dissection.

Afterwards, surgeons swept the internal sphincter muscle upwards with a gauze, in order to perform a safe excision of the hemorrhoidal

Correspondence to: $\mathrm{GA}$ Skarpas $\mathrm{MD}, \mathrm{PhD}$, Consultant Orthopaedic Surgeon/Lecturer of Anatomy, University of Athens, School of Nursing, 20 Papadiamandopoulou Str., Athens, Greece, Tel: +302107208100, +306942465650; E-mail: skarpasg@hotmail.com

Key words: anal canal anatomy, ligament of Parks, Trietz's muscle, conventional hemorrhoidectomy

Received: October 22, 2017; Accepted: November 15, 2017; Published: November 18, 2017 


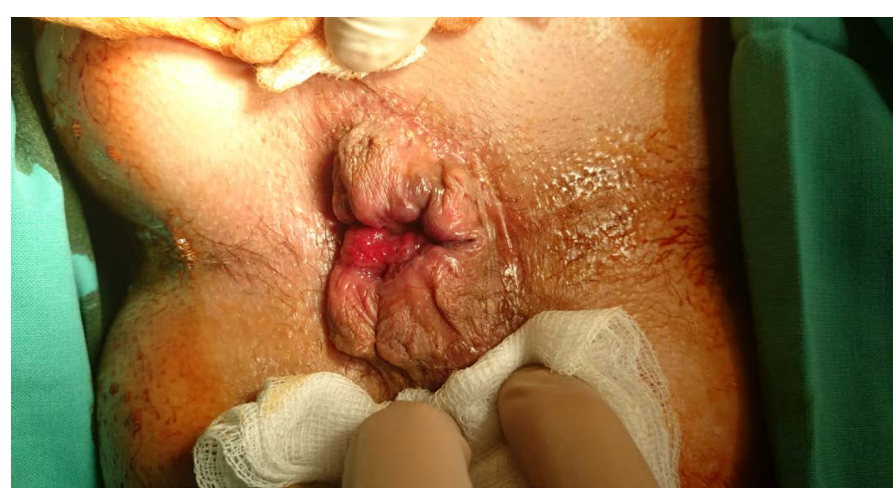

Figure 1. Prolapsing hemorrhoids.

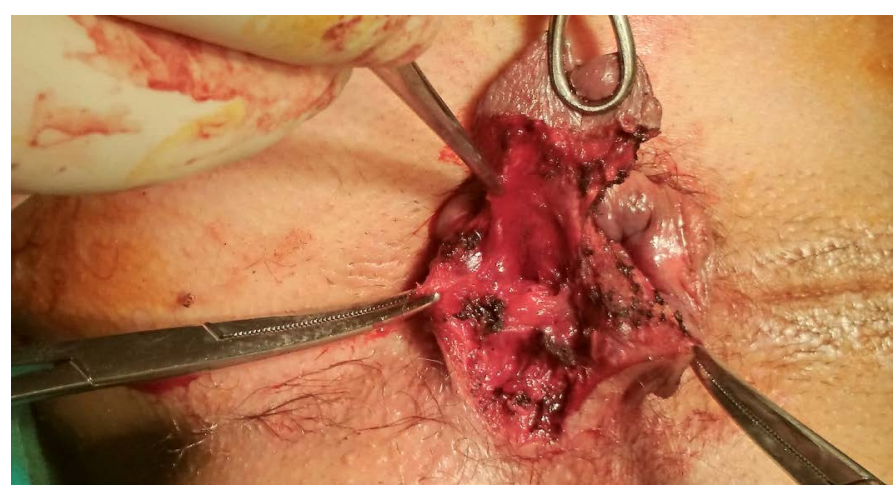

Figure 2. Dissecting in the submucosal plane in order to divide the Parks' ligament.

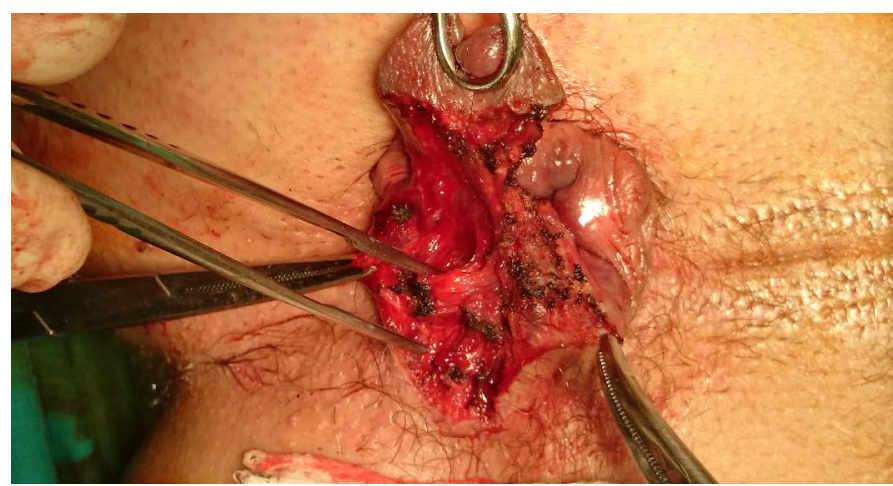

Figure 3. The identified mucosal ligament of Parks' pointed with the anatomic clamp.

pile without any potentiality of accidental muscular damage. Then, each pedicle was ligated with absorbable sutures. Additionally, the preserved mucocutaneous bridges were placed inside-out with a Chaput-Mayo grasper in order to remove the underlying remaining hemorrhoidal tissues. Finally, division of the pedicles with the cautery was performed with emphasis to careful hemostasis, for avoidance of postoperative bleeding, and a wound dressing was placed.

Ferguson's procedure: At first, a Hill - Ferguson retractor was inserted in the anus, a Kelly clamp was used to retract the hemorrhoid pedicle and an incision of elliptic shape around the hemorrhoidal pile was meticulously performed. Afterwards, a grasper was used for the elevation of the hemorrhoidal tissue and its bordering mucosa. The dissection was performed through the skin that is suited $1 \mathrm{~cm}$ above the dentate line of the anal canal. At the lateral border of the incision the Parks' ligament was identified and divided by the surgeons. Beneath this mucosal ligament, the white in colour internal sphincter was revealed and swept away with a gauze in order to remain intact during the procedure. Then, the borders of the incision's wound were elevated so that remnants of hemorrhoidal tissue could be dissected and suture closure of the rectal mucosa without tension could be achieved. The pedicle is suture-ligated with absorbable suture and then, the same suture is used for closure of the mucosa, the anoderm and the skin respectively. When surgeons encounter more than one hemorrhoid, each hemorrhoidal group is progressively treated in the same fashion.

Postoperative care: All patients were discharged with instructions the $2^{\text {nd }}$ or the $3^{\mathrm{d}}$ postoperative day. In fact, apart from the essential hygiene, the patients were advised by the clinicians to use a stool softener, since it reduces pain of the first defecation and prevents probable postoperative constipation. They were also consulted to increase their intake of dietary fibers, water and fluids and to decrease their intake of fat.

\section{Results}

From this total of 145 patients, 58 patients underwent Ferguson's $(40 \%)$ and 87 patients (60\%) underwent Milligan-Morgan's hemorrhoidectomy. The ligament of Parks (Trietz muscle) was identified to all patients and hemorrhoidectomy was successfully performed. More specifically, surgeons used the Parks' ligament as the lateral border of dissection, since the internal sphincter muscle is located just above it.

All the patients reported first defecation between the first and second postoperative day and none of the patients complained of fecal incontinence or urinary retention.

Nevertheless, in a female patient who underwent Milligan-Morgan hemorrhoidectomy, hemorrhage occurred the first postoperative day. Compression was sufficient to control the complication. Additionally, one patient who underwent the same procedure presented fever the first postoperative day. The infection was wed out with the administration of metronidazole and ceftriaxone.

Patients were also examined at 2 weeks after surgery, and none of them presented any complication. At 6 months thereafter only 127 patients proceeded for anoscopic inspection and had no any postoperative complication, such as prolapse, bleeding, anal stenosis or defecation pain.

\section{Discussion}

The armamentarium for hemorrhoidal disease includes several treatments such as Doppler-guided hemorrhoidal artery ligation [2,6] plication and stapled hemorrhoidopexy [4]. Nevertheless, for patients with grades III and IV hemorrhoids, surgery is considered as the gold standard among procedures. ${ }^{(3,6)}$

Surgery should be proposed to symptomatic patients after receiving an accurate history, performing clinical examination and colonoscopy or anoscopy for verification of the diagnosis of hemorrhoidal disease.

Although efficient, conventional hemorrhoidectomy is related to severe postoperative complications. Indeed, urinary retention, postoperative pain, anal incontinence, anal stenosis, bleeding and infection have been reported [1,7]. Such complications can be prevented by giving emphasis to the identification of the Parks' ligament (Trietz's muscle) as performed in the presented manuscript.

The ligament of Parks is consisted by muscular fibroplastic tissue that is located around the glands of the anus [8] and supports the hemorrhoidal plexus of the anal canal [2]. When the hemorrhoid cushions get enlarged and prolapsed, this mucosal ligament stretches 
and becomes irreversibly separated into pieces. In fact, it seems to present the most important anatomic structure in the formation of hemorrhoid cushions [9].

The Parks' ligament tethers the dentate line of the anal canal with the internal sphincter. It divides the anatomical space between the anal epithelium and the internal sphincter in two separate parts. Above the ligament, the submucous space is identified, containing the terminal part of the hemorrhoidal vessels. Below the ligament, lies the peri - anal space [10]. The Parks' ligament is cushion tissue in the anus, overwhelmed with blood vessels that could be defined as synonym of hemorrhoid tissue [9].

Either surgeons perform Milligan-Morgan [8] or Ferguson's hemorrhoidectomy [11] while dissecting the submucosal tissue, below the level of the dentate line they should identify the ligament of Parks. After dividing the mucosal ligament, surgeons acquire direct exposure of the internal sphincter. Subsequently, they can sweep away the muscular fibers so that they are able to dissect the hemorrhoids without damaging the muscle. Hence, the risk of anal incontinence can be minimized. Moreover, by emphasizing to the identification of Parks' ligament, excessive removal of the rectal mucosa and of the anoderm can be eliminated. Such strained excision of the anoderm and the rectal mucosa, may lead to avoidance of anal strictures.

Providing the ample sample of this study, the findings reveal that the Parks' ligament may guide surgeons during the operation, so that accidental damages and postoperative complications can be eliminated. Hence, surgeons should be fostered to pay particular attention to the identification of this key anatomical structure. Unfortunately, the limitation of the study is the absence of follow-up of 18 patients at six months, who did not proceed.

In conclusion, hemorrhoidectomy is an efficient and common therapeutic procedure for grades III and IV hemorrhoids but it is associated with many postoperative complications. Nevertheless, the ligament of Parks, which is a constantly identifiable key anatomical structure, may guide surgeons in a simple and reliable way through hemorrhoidectomy. Therefore, surgeons' thorough knowledge of the anatomy of the anal canal is a cornerstone of safe hemorrhoidectomy.

\section{Conflict of interest statement}

None.

\section{Funding}

There is no source of funding.

\section{Consent}

Written consent was provided from the patients for the publication of the case series and accompanying images.

\section{References}

1. Chen JS, You JF (2010) Current Status of Surgical Treatment for HemorrhoidsSystematic Review and Meta-analysis. Chang Gung Med J 33: 488-500. [Crossref]

2. Scheyer M, Antonietti E, Rollinger G, Mall H, Arnold S (2006) Doppler-guided hemorrhoidal artery ligation. The American Journal of Surgery 191: 89-93.

3. Li YD, Xu JH, Lin JJ, Zhu WF (2012) Excisional hemorrhoidal surgery and its effect on anal continence. World J Gastroenterol 18: 4059-4063. [Crossref]

4. Lohsiriwat $\mathrm{V}$ (2012) Hemorrhoids: from basic pathophysiology to clinical management World J Gastroenterol 18: 2009-2017. [CrossRef]

5. Bleday R, Pena JP, Rothenberger DA, Goldberg SM, Buls JG (1992) Symptomatic hemorrhoids: current incidence and complications of operative therapy. Dis Colon Rectum 35: 477-481. [Crossref]

6. Zagryadskiy EA, Gorelov SI (2008) Transanal Doppler-guided hemorrhoidal artery

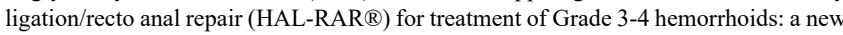
mini-invasive technology. Pelviperineology 27: 151-155.

7. Moult HP, Aubert M, De Parades V (2015) Classical treatment of hemorrhoids. J VisC Surg 152: S3-9. [Crossref]

8. Milligan ETC, Morgan CN (1937) Surgical anatomy of the canal anal and the operative treatment of haemorrhoids. Lancet 2: 1119-1124.

9. Yang HK (2014) The Pathophysiology of Hemorrhoids. In: Hemorrhoids. Verlag Berling Heidenberg: Springer; 14-24.

10. Parks AG (1958) Modern Concepts of the anatomy of the ano-rectal region. Postgrad Med J 34: 360-366. [Crossref]

11. Ferguson JA, Heaton JR (1959) Closed hemorrhoidectomy. Dis Colon Rectum 2: 176179. [CrossRef]

Copyright: (C2017 Mariolis-Sapsakos T. This is an open-access article distributed under the terms of the Creative Commons Attribution License, which permits unrestricted use, distribution, and reproduction in any medium, provided the original author and source are credited. 